\title{
Overall Comorbidity Score
}

National Cancer Institute

\section{Source}

National Cancer Institute. Overall Comorbidity Score. NCI Thesaurus. Code C150050.

Represents the severity grade of org an decompensation for the highest-ranked single comorbid ailment or, in the case in which there are two comorbid ailments in 2 different organ systems each rated as moderate organ decompensation, the overall score is severe. 